\title{
Factors Affecting Postoperative Agitation in Adults Following Functional Endoscopic Sinus Surgery: A Randomized, Double-Blinded Controlled Trial
}

\section{Hazem E Elsersy* and Ahmed Abdulazeez Ahmed \\ Lecturer of Anesthesia, Menoufya University, Egypt}

*Corresponding Author: Hazem Ezzat Elsersy, Lecturer of Anesthesia, Faculty of Medicine, Menofia University, Address: Zaki Shabanah Street, Alzanati tower, Shebin Alkom, Menofia, Egypt, Tel: 00201091096655; E-mail: hazelsersy@hotmail.com

\section{Research article \\ Volume 2 Issue 2}

Received Date: September 07, 2017

Published Date: September 12, 2017

\section{Abstract}

Background: We have noticed a high incidence of postoperative agitation (POA) following functional endoscopic sinus surgery (FESS), we reviewed our anesthesia protocol and designed this study. The present study investigated the possible contributing factors in the development of POA in adults, by avoiding these factors postoperative agitation can be minimized.

Methods: 140 ASA 1 and 2 patients were randomly allocated to either premedication group that received Phenergan 25 $\mathrm{mg}$ IM and atropine $0.5 \mathrm{mg}$ IM or none premedication group that received an equal volume of water for injection intramuscular injection. Hypotensive anesthesia was induced by nitroglycerin 5 to $20 \mathrm{mcg} / \mathrm{kg} / \mathrm{minute}$. In postoperative care unit (PACU), patients were assessed for both agitation and pain using Richmond agitation sedation scale and numerical rating scale respectively. Data were analyzed and compared between groups, $\mathrm{P}<0.05$ was considered significant.

Results: Agitation was more frequent in the premedication group; premedicated patients showed a higher incidence of agitation versus the control group $82 \%$ versus $35 \%$ respectively $\mathrm{P}<0.0001$.Postoperative agitation was more frequent in young ages, males, smokers and was correlated with pain. No correlation was found with BIS value or postoperative 02 .

Conclusion: premedication with atropine and Phenergan, Pain, smoking, young age, male gender was identified as risk factors for the development of postoperative agitation in adults following FESS.

Keywords: Agitation postoperative; Premedication atropine; Premedication Phenergan; Endoscopic sinus surgery 


\section{Anaesthesia \& Critical Care Medicine Journal}

\section{Introduction}

In our previous report on postoperative agitation in adults, we have investigated the role of magnesium sulfate as a potential treatment for postoperative agitations following functional endoscopic sinus surgery [1]. However, the high incidence of postoperative agitation in our protocol begs the search for the possible contributing factors to this phenomenon. Postoperative agitation although short-lived it could be harmful to both patients and recovery staff $[2,3]$. An agitated patient has the potential for self-injury by removing intravenous cannulas, tubes, oxygen masks, and nasal packs. Furthermore, very agitated patients can pose an immediate danger to operating room staff [4]. In the last few years, and during our work with patients subjected to endoscopic sinus surgery, we have observed a high incidence of postoperative agitation that was not terminated by administration of narcotic analgesics.In the postoperative care unit, we noticed some agitated patients forcibly removing their venous and arterial cannulas, Oxygen masksresulting in bleeding and hypoxia. We also recorded bruises and ecchymosis from the restraints of agitated patients.These complications have forced us to review our anesthesia protocol and implement a study to identify the risk factors that might account for this phenomenon. Kim, et al. [5] reported that postoperative agitation following nasal surgery could occur due to several factors; young age, smoking, sevoflurane anesthesia, postoperative pain, the presence of a tracheal tube or a urinary catheter. Some of the drugs that are used in anesthetic pre-medication have been shown to increase excitation and agitations [6]. Amongst these anticholinergic and antihistaminic drugs are incriminated [7]. We have sought this study to explore whether the used Atropine and Phenergan have contributed to the high incidence of postoperative agitation. The other aim is to discover the other contributing factors that are responsible for postoperative agitation in adults following functional endoscopic sinus surgery.

\section{Methods}

\section{Patients and Design}

This study was approved by the Menofia University ethics committee on January $6^{\text {th }}$ 2014. 140 American society of anesthesiologist- physical status I and II patients of both genderswere enrolled in the study, then written consent was obtained from each participant.
Inclusion criteria were age between 18 and 60 years of both sexes, ASA I and II statuses, and patient acceptance to participate in the study. Exclusion criteria were hypertensive patients, cardiac ischemia, cerebrovascular insufficiency, neuromuscular diseases, pregnancy, prolonged treatment with calcium channel blockers, diabetic neuropathy. Patients were randomized by a research randomizer computer system and were assigned to either Pre-medication (Group A) or the control (Group B) by central randomization through phone calls to an anesthesia technician who was not participating in the study; he was furnished patient ID, body weight, and group allocation and was tasked to prepare colorless coded solutions contained in transparent syringes. Group A patients were premedicated by atropine sulfate Boden Centra limited $(0.5 \mathrm{mg} / \mathrm{ml}) 0.5 \mathrm{mg} \mathrm{IM}$ and Phenergan (Promethazine $\mathrm{HCl}$, Lab Renaudine, France) as a sedative and antiemetic $25 \mathrm{mg}$ IM one hour before entering the operation theater. Group B patients were given an equal volume of plain water injection intramuscularly. All patients underwent functional endoscopic sinus surgery at Menofia University Hospitals ENT room. For the purpose of estimating the incidence of agitation in relation to age,all patients were classified into 2 categories depending on their ages. Category one included age range from 18 to 30 (Cat 1), category two included age range from 31 and up (Cat 2). The smoking history was taken; smokers were ranked according to the duration and daily consumption of cigarettes into 4 categories $0,1,2$ and 3 . A smoking index was implemented by multiplying the number of cigarettes per day by the smoking years, and the resulted number was graded on afour-point scale: nonsmoker, light, heavy and massive smoker as follows; $0=0,1=1: 200,2=201: 400$ and $3=401: 600$. Below the smoking index variable, each patient was assigned a number depending on his smoking status, $0=$ nonsmoker, $1=$ light smoker, $2=$ heavy smoker, and $3=$ massive smoker.

\section{Anesthesia Technique}

A large bore 18-gauge intravenous cannula was inserted, all patients underwent general endotracheal anesthesia and were anesthetized by Propofol $2.5 \mathrm{mg} / \mathrm{kg}$, Fentanyl $1 \mathrm{mcg} / \mathrm{kg}$. Atracurium $0.5 \mathrm{mg} / \mathrm{kg}$ was given to facilitate intubation and ventilation. Anesthesia was maintained by sevoflurane $2 \%$ in $50 \%$ air and Oxygen. All patients were monitored by standard monitors including, the pulseoximeter, non-invasive blood pressure, end-tidal $\mathrm{CO}_{2}$, and electrocardiogram. Moreover, an arterial line was inserted, and invasive blood pressure was monitored 


\section{Anaesthesia \& Critical Care Medicine Journal}

throughout the procedure. Sevoflurane, $\mathrm{O}_{2}$ percentage concentrations were monitored throughout the procedure. Anesthetic depth was monitored using bispectral index (Aspect XP, USA) to exclude awareness as a contributing factor to the development of agitation. Hypotension was induced by infusion of nitroglycerine $(5 \mathrm{mg} / \mathrm{ml}$ Hospira UK Limited) at a rate of 5 to $20 \mathrm{mcg} / \mathrm{kg}$ /minute titrated to target mean blood pressure of $55 \pm 5$. The purpose of hypotensive anesthesia was to reduce bleeding and improve the surgical field. After the onset of hypotension Mean arterial pressure and Bispectral index were recordedsimultaneously every 10 minutes. The medians of the obtained readings were calculated for each patient and represented the Med. MAP and Med. BIS respectively. Hypotensive anesthesia was maintained throughout the surgical procedure. After the conclusion of surgery, patients were given ephedrine hydrochloride (Martin DalePharmaceuticals) $10 \mathrm{mg}$ IV to restore the mean arterial pressure back to the preoperative value. Upon attaining spontaneous breathing patients were given reversal to muscle relaxants and extubated in a wake mode. Upon recovery,one gram Paracetamol (Perfalgan, Bristol Mayers Squeeb) was given intravenously to each patient.

\section{Patient Assessment}

Patients were transferred to PACU for observation and assessment for agitation and pain. Upon arrival to PACU, a pulse oximeter and NIBP were attached to the patient. Discharge criteria from PACU were stable vital signs, pain score less than or equal to 2 , no nausea or vomiting, calm and alert patient. Patient age, body weight, gender, and vital signs were recorded and compared between groups.

\section{Assessment of agitation}

Agitation was defined as a purposeless excessive motor activity and/ or inconclusive sounds or aggressive behavior of the patient with no response to commands. Postoperative agitation was assessed in the PACU by a blinded observer using the Richmond AgitationScale (RASS) as follows:

$0=$ Alert and calm

$+1=$ Restless; Anxious and /or apprehensive but movements not aggressive.

$+2=$ agitated; Frequent non-purposeful movement.

$+3=$ very agitated; Pulls on or removes the tubes or catheters or has aggressive behavior toward staff.

$+4=$ combative; overly combative or violent.

Total agitation was defined as the algebraic sum of agitation values observed at the measured time points, and this was used to correlate agitation with other variables. The range of agitations was $0=$ no agitation to $20=$ severe persistent agitation .

\section{Assessment of pain}

Pain scores were assessed using numerical rating scalewhere $0=$ no pain and 10 is the worst imaginable pain as rated by the patient. A blinded team member assessed pain after evaluation of last agitation point (30 minutes of recovery). Patient that exhibited no agitation were also evaluated for pain after 30 minutesof recovery. Pethidine was used as rescue analgesic to ameliorate pain. The use of pethidine was intendedafter evaluation of agitation and assessment of pain to avoid confounders to the outcome measurement. Pethidine was given in $25 \mathrm{mg}$ increments every 10 minutes until pain score $\leq 2$.

\section{Statistical analysis}

Power analysis was done based on our pilot study comparing the frequency of agitation 10minutes postoperatively as a primary determinant of sample size. Using means difference between the two groups (effect size) of $25 \%$, given $\alpha$ probability $=0.05$, power $95 \%$ revealed a total sample size of 132 is required, 66 per group to detect $25 \% \%$ reduction in postoperative agitation. We increased the total sample size to 140 to avoid a drop-in sample size due to possible exclusions. Descriptive statistics were used to represent patient characteristics. Although the Richmond Agitation Sedation Scale values are ordinal variables, we have used 5 defined points that can be treated as a continuous variable [8]. Continuous variables were tested for normality and were represented by means or medians as appropriate; categorical data were represented by percent of the total. All agitation points were compared by Man-Whitney U test, Medians of pain scores were compared by Mood'stest.Correlation between agitation and other variables was evaluated by Spearman correlation test and Chi-square test. The coefficients of determination $\left(\mathrm{R}^{2}\right)$ for pain, BIS, smoking index and PACU stay were estimated by ANCOVA. $\mathrm{P}<0.05$ was considered significant.

\section{Results}

Figure 1 shows, patient enrollment, and randomization process. 140 patients were randomly allocatedtopremedication group $(\mathrm{N}=70)$ or the control group $(\mathrm{N}=70) .3$ patients were excluded from the final analysis because of atypical hypotension 2in pre- 


\section{Anaesthesia \& Critical Care Medicine Journal}

medication and 1 in the control group. 137 patients were analyzed, 68 in pre-medication and 69 in the control

group. There was no difference in patient characteristics (Table1).

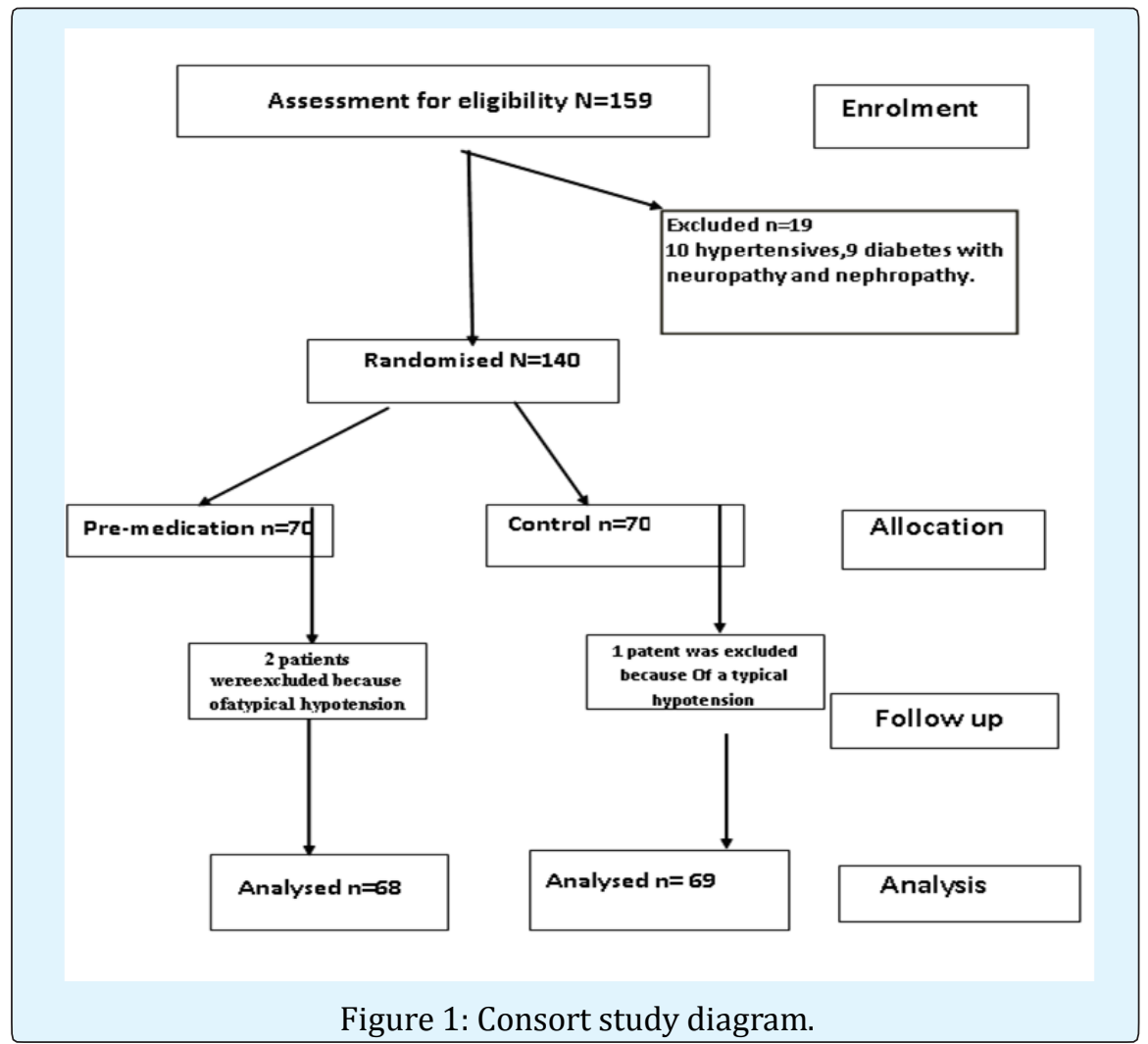

NB:- Atypical hypotension is rapid swinging of blood mean) pressure (too much decrease and increase with unstable

\begin{tabular}{|c|c|c|c|}
\hline Variable & Control G & Premedication G & P Value \\
\hline Number & 68 & 69 & 0.1 \\
\hline Age(years) & $35 \pm 10$ & $33 \pm 12$ & 0.06 \\
\hline $\mathrm{BMI}(\mathrm{Kg} / \mathrm{m} 2)$ & $30.6 \pm 3$ & $31 \pm 4$ & 0.05 \\
\hline $\mathrm{ETCO}_{2}(\mathrm{~mm} \mathrm{Hg})$ & $36 \pm 3$ & $35 \pm 4$ & 0.05 \\
\hline $\mathrm{BIS}$ value & $40 \pm 3$ & $39 \pm 2$ & 0.9 \\
\hline Recovery SPO & $99 \pm 0.9$ & $99 \pm 0.8$ & 0.9 \\
\hline Pre-MAP (mm Hg) & $89 \pm 4$ & $88,6 \pm 4$ & 0.5 \\
\hline Med MAP (mmHg) & $53 \pm 3$ & $54 \pm 4$ & 0.9 \\
\hline Rec. MAP (mmHg) & $89 \pm 4$ & $88.6 \pm 4$ & \\
\hline Gender M/F & $29 / 40$ & $30 / 38$ & \\
\hline
\end{tabular}

Table 1: Patient characteristics.

Table 1 reveals the patient characteristics, bispectral index, end-tidal carbon dioxide, recovery oxygen saturation and mean arterial blood pressure in both groups. There was no statistical difference between the two groups in these parameters. $\mathrm{BMI}=$ body mass index, $\mathrm{G}=$ group, $\mathrm{ETCO}_{2}=$ end-tidal carbon dioxide, Recovery $\mathrm{SPO}_{2}=0$ oxygen saturation measured at recovery room, $\mathrm{M} / \mathrm{F}=$ Male and female. 


\section{Anaesthesia \& Critical Care Medicine Journal}

The incidence of agitation among all patient was found to be 68\%; Premedicated patients showed a higher incidence of agitation versus the control group 82\% versus $35 \%$ respectively $\mathrm{P}<0.0001$. Young patients showed higher incidence and more severe agitation than older patients did. Cat1 patients $(n=68)$ showed an $85 \%$ incidence of postoperative agitation (POA), Cat2 $(n=69)$ showed an 35\% incidence ofPOA. There was a correlation between age and agitation severity as measured by Pearson analysis (correlation coefficient $=0.0 .75$ ). Females showed a lower incidence of postoperative agitation; the study included 77 males and 59 females, agitation was evident in 67 males and 10females, which represent $87 \% \%$ versus $22 \%$ in males and femalesrespectively. Chisquare test revealed an association between gender and agitation with the correlation coefficient $0.725, \mathrm{P}<0.0001$ (Table 2). In the total sample size, there were 95nonsmokers, and 42 smokers, the incidence of agitation was found to be $88 \%$ among smokers versus $\% 44 \%$ for nonsmokers $\mathrm{P}<0.0001$. There was no statistical difference in pain score between the two studied groups, pain scores of $4.7 \pm 1.2$ versus $4.4 \pm 1.3$ in pre-medication and the control group respectively, $\mathrm{P}=0.207$ however, there was a correlation between postoperative pain, and agitation (Table2).

There was no significant correlation between agitation

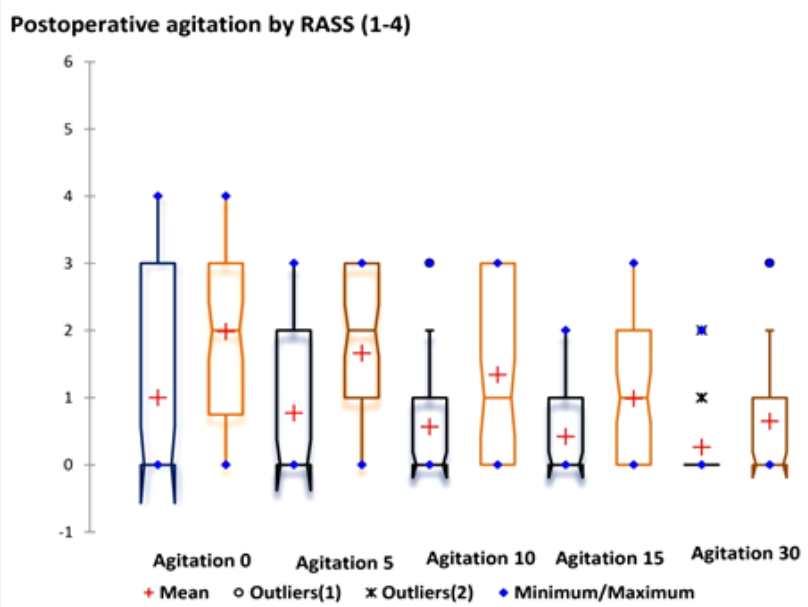

Figure2: postoperative agitation in following FESS.

Figure 2: Postoperative agitation in both premedication (drown bars) and the control (blue bars) groups measured by Richmond agitation scale at $0,5 \mathrm{~m} \mathrm{10,15}$ and 30 minutes of recovery the red cross represents mean; the middle line represents the medium, the blue dots represents the outliers, and the notches represent the confident interval around the median RASS=Richmond agitation sedation scale.

and either postoperative 02 saturation or BIS (Table 2).

\begin{tabular}{|c|c|c|c|c|c|c|}
\hline Test & Smoking index & SPO2 & Pain & BIS & age & gender \\
\hline Spearman coefficient of determination & 0.578 & 0.1 & 0.25 & 0.01 & 0.43 & \\
\hline ANCOVA R $^{2}$ & 0.3 & 0.08 & 0.2 & 0.01 & 0.34 & \\
\hline Chi square & 0.671 & 0.06 & 0.389 & 0.01 & 0.75 & 0.72 \\
\hline P value & $<0.0001$ & 0.07 & 0.001 & 0.333 & $<0.0001$ & $<0.0001$ \\
\hline
\end{tabular}

Table 2: Correlation coefficients between variables and total agitation.

Table 2 Shows the correlation between Smoking, Oxygen saturation, Postoperative pain, Intraoperative anesthetic depth, age, gender and the total agitation. There was a correlation between total agitation and Smoking, pain, age, and gender but no correlation between total agitation and recovery Oxygen saturation or Bispectral index.

\section{Discussion}

The main finding of the present study is that premedication with atropine and Phenergan increased the occurrence and severity of postoperative agitation in adults following functional endoscopic sinus surgery. postoperative agitation in adults has not been adequately studied, few studies have reported the occurrence postoperative agitation in adult patients following nasal surgery $[9,10]$. A plenty of medications have been shown to increase delirium and excitations, [11] anticholinergics and antihistaminic are of particular concern as these drugs are routinely used in the pre-anesthetic medications [12]. The results of the present study reveal that pre-medication with both Atropine (an anticholinergic) and Phenergan (an antihistamine) substantially increase the occurrence of postoperative agitation following FESS. Because both Atropine and Phenergan cross the blood brain barrier, they may interact to cause central excitation that is noted as 


\section{Anaesthesia \& Critical Care Medicine Journal}

postoperative agitation. These agitations could be minimized by the use of a quaternary ammonium anticholinergic like glycopyrrolate as an Atropinesubstitute. Some postoperative events might provoke agitation, postoperative pain [13] and hypoxia [14], are major confounders that may cause or result from agitation. While hypoxia and pain can cause agitation, an agitated patient can remove his 02 mask or injure himself resulting in more hypoxia and pain. In our study, because none of the patients showed are duction in recovery oxygensaturation, there was no correlation between agitation and the recovery oxygen saturation; this would exclude hypoxia as a confounding factor for postoperative agitation. In the present study,There was a correlation between agitation and postoperative pain, indicating that pain was a contributing factor in the development of agitation. Inspite of that, pain cannot be considered the only cause of POA because, in the present study, the postoperative pain was equal in both groups while premedication group displayed a significantly higher agitation.In addition, the correlation coefficient between pain and agitation was found to be 0.25 , and this denotes that pain can explain $25 \%$ only of the agitation. Consistent with this, Weldon, et al. reported that emergence agitation could occur even after adequate pain treatment or after procedures that are not associated with pain [15]. Postoperative agitation in adults was reported mainly in geriatric population [16,17], Surprisingly more frequent and more severe agitation were detected among 18 to 30 years old group of patients. We expected the reverse to occur as delirium was almost recorded in geriatric patients $[18,19]$. It is apparent that this type of POA represents a separate entity than those noticed for geriatric populations. Although both POA and delirium are manifested by patient confusion and excitation, it appears that the etiology and predisposing factors are different. POA in our study mimics those displayed by children anesthetized with sevoflurane rather than that shown in geriatric populations. Agitation was more prominent among smokers, the matter that raises the question whether the increased agitation is due to nicotine effects or nicotine withdrawal? Nicotine withdrawal during the fasting period may cause restlessness and irritability. Nicotine withdrawal has been shown to be associated with a negative emotional state, including anxiety and the perception of increased stress $[20,21]$. withdrawal symptoms include irritability, depressed mood, restlessness, anxiety, difficulty concentrating and insomnia [20]. It is possible that nicotine abstinence during the fasting period ( 8 hours), duration of surgery average (2) hours have accounted for the increased agitation among smokers. Further studies are required to clarify this issue. Agitation was more frequent and severe among males than females; one possible explanation is the hormonal difference between both sexes. Interestingly, testosterone hasbeen shown to decline with advancing age [22]. So it is possible that higher testosterone level in young males has contributed to increased agitation in this group. Further studies are clearly required to address this issue. Intraoperative awareness has not been detected during our study as measured by the bispectral index. Therefore, in our protocol, we can exclude awareness as a risk factor for postoperative agitation. Nevertheless, the accuracy of BIS as a tool to measure intraoperative awareness is controversial [23-28]. It remains possible that some degree of awareness has occurred during hypotension where inhalational anesthetic concentration was reduced. There are no data on the effect of hypotension on BIS value. Furthermore, it is not clear if this level of hypotension has suppressed brain electrical activity to yield a significant reduction in BIS recording. It remains possible that an unknown effect of hypotension has decreased BIS value. This reduction Was counteracted by some degree of awareness during hypotension when the inhalational anesthetic concentration was reduced, so the net value was equal. Emergence agitation is costly in several ways, in terms of morbidity, in human resources and on the financial level [29]. It is well-known that personnel costs account for the majority of PACU costs $[30,31]$. The agitated patient requires 4-6 recovery nurses to control his movement and apply restraints, take care of monitoring. In our study agitated patients removed oxygen masks, IV cannulas, nasal pack, pulse oximeter probes several times before being quiet, the matter that shifted attention to this particular patient, rendered many recovery staff busy at the expense of the other patients. We acknowledge that our study has some limitations. First,we did not study the effect of hypotension on agitation because all patients were subjected to induced hypotension to reduce bleeding and improve the surgical field. Second, the role of the nasal pack in the development of POA was not investigated. Further studies are needed to answer these questions.

\section{Conclusion}

a. Premedication with Atropine and Phenergan increase the incidence of postoperative agitation. Avoiding premedication or the use of their substitutes might reduce the incidence of postoperative agitation. 


\section{Anaesthesia \& Critical Care Medicine Journal}

b. Postoperative pain, smoking, young age, male gender were identified as risk factors for postoperative agitations following FESS.

- Author Contribution: Hazem E. Elsersy: Idea, experimental design, patient recruitment, randomization, data analysis, manuscript writing. Ahmed A A.: experimental design, data analysis.

- Acknowledgment: The authors thank our anesthesia technicians and nurses who helped very much in outcome assessment.

- Conflict of interest: none declared

- Funding: -This research was funded by Menoufya University; none of the authors have any personal financial interest in this research.

\section{References}

1. Elsersy HE1, Metyas MC, Elfeky HA, Hassan AA (2017) Intraoperative magnesium sulphate decreases agitation and pain in patients undergoing functional endoscopic surgery A randomised double-blind study. Eur J Anaesthesiol 34(10): 658-664.

2. Rose DK (1996) Recovery room problems or problems in the PACU. Can J Anaesth 43: 116-128.

3. Pieters BJ, Penn E, Nicklaus P, Bruegger D, Meht B, et al. (2010) Emergence delirium and postoperative pain in children undergoing adenotonsillectomy: a comparison of propofol vs sevoflurane anesthesia . Pediatr Anesth 20(10): 944-950.

4. Kuratani N, Oi Y (2008) Greater incidence of emergence agitation in children after sevoflurane anesthesia as compared with halothane: a metaanalysis of randomized controlled trials. Anesthesiology 109(2): 225-232.

5. Hyo-Jin Kim, Duk-Kyung Kim, Hyo-Yeol Kim, JinKyoung Kim, Seung-Won Choi (2015) Risk factors of Emergence Agitation in Adults Undergoing General Anesthesia for Nasal Surgery. Clin Exp Otorhinolaryngol 8(1): 46-51.

6. Moore AR, O'Keefe ST (1999) Drug-induced cognitive impairment in the elderly. Drugs Aging 15(1): 15-28.

7. Han L, McCusker J, Cole M, Abrahamowicz M, Primeau F (2001) Use of medications with anticholinergic effect predicts clinical severity of delirium symptoms in older medical inpatients. Arch Intern Med 161(8): 1099-1105.

8. Curtis N, Mark S, Mary J, Gretchen M, Pam V, et al. (2002) The Richmond Agitation-Sedation Scale Validity and reliability in Adult Intensive Care Unit Patients. Am J Respir Crit Care Med 166(10): 13381344.

9. Kim SY, Kim JM, Lee JH, Song BM, Ko BN (2013) Efficacy of intraoperative dexmedetomidine infusion on emergence agitation and quality of recovery after nasal surgery. Br J Anaeth 111(2): 222-228.

10. Daihua Yu, Wei Chai, Xude Sun, Linong Yao (2010) Emergence agitation in adults: risk factors in 2,000 patients. Can J Anesth 57(9): 843-848.

11. Alagiakrishnan K, Wiens CA (2004) An approach to drug induced delirium in the elderly. Postgrad Med J 80(945): 388-393.

12. Clegg A, Young JB (2011) Which medications to avoid in people at risk of delirium: a systematic review. Age and Ageing 40(1): 23-29.

13. Veyckemans F (2001) Excitation phenomena during sevoflurane anesthesia in children. Curr Opin Anaesthesiol 14(3): 339-343.

14. Jakub K, Maciej K, Maciej B, Wojciech F, Piotr O, et al. (2010) Incidence and predictors of delirium after cardiac surgery: Results from the IPDACS Study. $J$ seR mosohcysP69)2:(179-185.

15. Weldon BC, Bell M, Craddock T (2004) The effect of caudal analgesia on emergence agitation in children after sevoflurane versus halothane anesthesia. Anesth Analg 98(2): 321-326.

16. Marcantonio ER, Juarez G, Goldman L, Mangione CM, Ludwig LE, et al. (1994) The relationship of postoperative delirium with psychoactive medications. JAMA 272(19): 1518-1522.

17. Lynch EP, Lazor MA, Gellis JE, Orav J, Goldman L, et al. (1998) The impact of postoperative pain on the development of postoperative delirium. Anesth Analg 86(4): 781-785. 


\section{Anaesthesia \& Critical Care Medicine Journal}

18. Thomas NR, Bin E (2008) Postoperative delirium in the elderly: diagnosis and management. Clin Interv Aging 3(2): 351-355.

19. Dasgupta M, Dumbrill AC (2006) Preoperative assessment of delirium after noncardiac surgery: a systemic review. J Am Geriatr soci 54(10): 15781589.

20. Nestler EJ (2005) Is there a common molecular pathway for addiction? Nat Neurosci 8(11): 14451449.

21. Benowitz NL (2009) Pharmacology of nicotine: Addiction, smoking-induced disease, and therapeutics. Annu Rev Pharmacol Toxicol 49: 57-71.

22. Travison TG, Araujo AB, Kupelian V, O'Donnell AB, McKinlay JB (2007) The Relative Contributions of Aging, Health, and Lifestyle Factors to Serum Testosterone Decline in Men. J Clin Endocrinol Metab 92(2): 549-555.

23. Bruhn J, Kreuer S, Bischoff P, Kessler P, Schmidt GN, et al. (2005) Bispectral index and A-line AAI index as guidance for desflurane-remifentanil anesthesia compared with a standard practice group: a multicenter study. Br J Anaesth 94(1): 63-69.

24. Bruhn J, Myles PS, Sneyd R, Struys MM (2006) Depth of anesthesia monitoring: what's available, what's validated and what's next? Br J Anaesth 97(1): 85-94.

25. Avidan MS, Zhang L, Burnside BA, Finkel KJ, Searleman AC, et al. (2008) Anesthesia Awareness and the Bispectral Index. N Engl J Med 358(11): 10971108.
26. Bruhn J, Bouillon TW, Radulescu L, Hoeft A, Bertaccini E, et al. (2003) Correlation of approximate entropy, bispectral index, and spectral edge frequency 95 (SEF95) with clinical signs of anesthetic depth' during co-administration of Propofol and remifentanil. Anesthesiology 98(3): 621-627.

27. Kearse I, Rosow C, Zaslavsky A, Connors P, Dershwitz $M$, et al. (1998) Bispectral analysis of the electroencephalogram predicts conscious processing of information during Propofol sedation and hypnosis. Anesthesiology 88(1): 25-34.

28. Leslie K, Sessler DI, Schroeder M, Walters K (1995) Propofol blood concentration and the Bispectral Index predict suppression of learning during Propofol/epidural anesthesia in volunteers. Anesth Analg 81(6): 1269-1274.

29. Lepouse C, Lautner CA, Liu L, Gomis P, Leon A (2006) Emergence delirium in adults in the postanesthesia care unit. Br J Anaeth 96(6): 747-753.

30. Dexter F, Tinker JH (1995) Analysis of strategies to decrease postanesthesia care unit costs. Anesthesiology 82(1): 94-101.

31. Dexter F, Penning DH, Traub RD (2001) Statistical analysis by Monte-Carlo simulation of the impact of administrative and medical delays in discharge from the post anesthesia care unit on total patient care hours. Anesth Analg 92(5): 1222-1225.

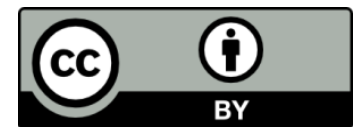

Hazem E Elsersy and Ahmed Abdulazeez Ahmed. Factors Affecting Postoperative Agitation in Adults Following Functional Endoscopic Sinus Surgery: A Randomized, Double-Blinded Controlled Trial. Anaesth Critic Care Med J 2017, 2(2): 000123. 